\title{
考虑附加力影响的弹性体远程火箭弹弹道分析
}

\author{
魏 钊 1,2 龙腾 1,2 李怀建 ${ }^{1,2}$ 王 祝 ${ }^{1,2}$ 刘 莉 1,2 \\ (1. 北京理工大学宇航学院 北京 100081 ; \\ 2. 北京理工大学飞行器动力学与控制教育部重点实验室 北京 100081)
}

\begin{abstract}
摘要: 远程火箭弹具有长细比大、射程远的特点, 为提高弹道分析精度, 有必要充分考虑气动弹性和其它附加力的影响。以 远程火箭弹非线性运动方程为基础, 考虑气动弹性效应, 分别采用细长体理论和气动导数法计算弹身和弹翼舵面产生的非定 常气动力, 并考虑地球自转和发动机燃料变化所引起的附加力以及地球曲率的影响, 建立弹性体远程火箭弹六自由度运动模 型。通过弹道仿真, 对比分析气动弹性、附加力和结构刚度对远程火箭弹脱靰量的影响。仿真对比结果表明, 气动弹性和附 加力都对远程火箭弹脱靶量产生影响, 且附加力与气动弹性高度耦合, 在考虑气动弹性效应的前提下, 附加力对远程火箭弹 脱靶量的影响被显著放大, 因此在全弹道仿真分析中必须同时考虑气动弹性和附加力的耦合影响; 弹体结构刚度越小, 远程 火箭弹的脱靶量越大, 在远程火箭弹初步设计中, 应保证弹体结构刚度以确保命中精度。
\end{abstract}

关键词: 远程火箭弹; 气动弹性; 附加力; 结构刚度; 全弹道仿真; 脱靶量

中图分类号: TJ71

\section{Trajectory Analysis of Aeroelastic Long-range Rockets Considering Subsidiary Load Effect}

\author{
WEI Zhao ${ }^{1,2}$ LONG Teng ${ }^{1,2}$ LI Huaijian ${ }^{1,2}$ WANG Zhu ${ }^{1,2}$ LIU Li ${ }^{1,2}$ \\ (1. School of Aerospace Engineering, Beijing Institute of Technology, Beijing 100081; \\ 2. Key Laboratory of Dynamics and Control of Flight Vehicle of Ministry of Education, \\ Beijing Institute of Technology, Beijing 100081)
}

\begin{abstract}
Long-range rockets have the characteristics of large slenderness ratio and long range. To improve the accuracy of trajectory simulation, it is necessary to consider aeroelasticity and subsidiary load effect. Based on the nonlinear motion equation of the rockets, the effect of aeroelasticity is is incorporated in the trajectory simulations. The unsteady aerodynamic loads of the body, wings and rudders are calculated by slender body theory and aerodynamic derivative method, respectively. Besides, the six-degree-of-freedom flight dynamics model considering the curvature and the subsidiary loads generated by Earth rotation and fuel mass change is built. Then, the effect of aeroelasticity, subsidiary loads and structural stiffness on rocket miss distance is analyzed through the trajectory simulations. Simulation results show that aeroelasticity and subsidiary loads have a great impact on the miss distance of the rocket. In addition, the subsidiary loads are highly coupled with aeroelasticity and the effect of the subsidiary loads on miss distance of the rocket is significantly amplified with the consideration of aeroelasticity effect. Thus, it is necessary to consider the coupling effect of aeroelasticity and subsidiary loads simultaneously in whole trajectory simulation analysis. Furthermore, when the rocket structural stiffness becomes insufficient, the miss distance of the rocket increases greatly. Therefore, sufficient structural stiffness is essential for improving the hit precision in rocket preliminary design.
\end{abstract}

Key words: long-range rocket; aeroelasticity; subsidiary load; structural stiffness; trajectory simulation; miss distance

\section{0 前言}

武器装备的发展和战场需求的提高, 对远程火 箭弹的命中精度和效益性提出了更高的要求 ${ }^{[1-2]}$ 。远

20180425 收到初稿, 20190214 收到修改稿
程火箭弹具有长细比大、射程远和飞行速度高等特 点, 且飞行特性和命中精度受刚体运动、弹性振动、 气动力和控制系统之间耦合的影响 ${ }^{[3]}$, 用刚体模型 进行弹道仿真会产生较大误差, 因此气动弹性是火 箭弹弹道分析中的重要一环 ${ }^{[4-6]}$ 。另外, 当火箭弹射 程超过 $100 \mathrm{~km}$ 时, 若把地球当作平面大地, 同时忽 略由于地球自转和发动机燃料变化产生的附加力, 
则不能真实反映作战打击情况 ${ }^{[7]}$ 。因此, 针对远程 火箭弹六自由度全弹道分析问题, 有必要在弹道仿 真中考虑气动弹性、地球曲率与自转等因素的影响。

针对大长细比弹箭弹道分析问题, 杨䎦明等 ${ }^{[8]}$ 在 MATLAB/Simulink 环境下, 采用全弹道模块化建 模的方法, 完成了子导弹的全弹道数字仿真, 但该 弹道模型并没有考虑气动弹性和地球自转等产生的 附加力的影响。杨希祥等 ${ }^{[9]}$ 针对小型多级固体运载 火箭, 采用侧喷流和栅格舵控制技术, 开展了六自 由度弹道仿真研究, 提高了固体运载火箭的运载能 力, 文献中的火箭在仿真过程中考虑了地球自转等 产生的附加力的影响, 但没有考虑火箭由于弹性变 形而产生的弹性效应。刘莉等 ${ }^{[10]}$ 建立了考虑气动弹 性的三自由度导弹弹道分析模型, 研究分析了弹性 对导弹在半实物仿真过程中的影响, 但是该模型忽 略了侧向运动。YAO 等 ${ }^{[11]}$ 建立了平面地表模型下考 虑气动弹性的六自由度弹道分析模型, 并研究分析 了弹性特性对导弹命中精度的影响; SCHMIDT 等 ${ }^{[12]}$ 在刚体飞行器动力学模型的基础上, 加入弹性振动 方程并完成了仿真, 并分析了数字延时等约束对仿 真结果的影响。以上两篇文献没有考虑地球的曲率 以及自转而产生的附加力, 对于弹性体远程火箭弹 的弹道仿真而言, 会产生较大误差。DU 等 ${ }^{[13]}$ 建立 了椭球地表模型下“战神”弹性体火箭弹的六自由度 弹道分析模型, 并完成了仿真, 但该模型没有考虑由 于地球自转以及发动机燃料变化而产生的附加力。

根据文献调研, 前人已经在分别考虑气动弹性、 地球自转等因素的远程火箭弹弹道建模与分析方面 开展了一系列研究。但是, 尚未发现综合考虑气动 弹性、地球自转和发动机燃料变化产生的附加力以 及地球曲率等多种因素影响的火箭弹弹道建模与分 析研究工作。对此, 本文建立了一套完整的六自由 度弹性体远程火箭弹全弹道分析模型, 考虑了气动 弹性、地球自转和发动机燃料变化产生的附加力以 及地球曲率的影响, 完成了弹道仿真, 并对比分析 了气动弹性、地球自转以及燃料变化产生的附加力 和结构刚度对火箭弹脱靶量的影响。

\section{1 远程火箭弹飞行仿真建模}

远程火箭弹弹道仿真的总体框图如图 1 所示。 由于弹性效应的影响, 火箭弹的弹性变形会造成附 加攻角、侧滑角和舵偏角的产生, 推力的方向也会 发生变化, 此外, 弹性振动会造成敏感元件测量有 偏差。图中颜色加深的部分表示弹性体火箭弹弹道 仿真与刚体火箭弹弹道仿真存在显著差异的环节。

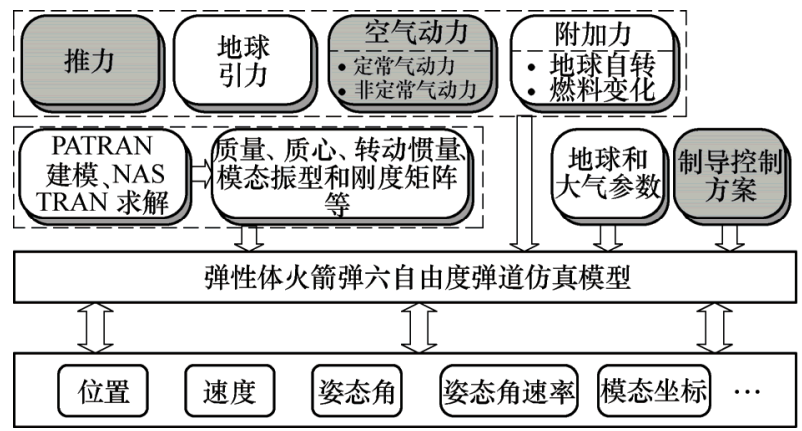

图 1 远程火箭弹弹道仿真总体框图

\section{1 远程火箭弹模态分析}

在火箭弹结构有限元建模过程中, 弹身采用梁 模型构建 ${ }^{[14-15]}$, 把弹翼、舵面和燃料当作非结构单 元加在相应位置的有限元节点上, 将燃料等规则体 均匀分布在发动机位置的每个有限元节点上, 将弹 翼和舵面等不规则体按比例进行质量配置，火箭弹 结构示意图如图 2 所示。

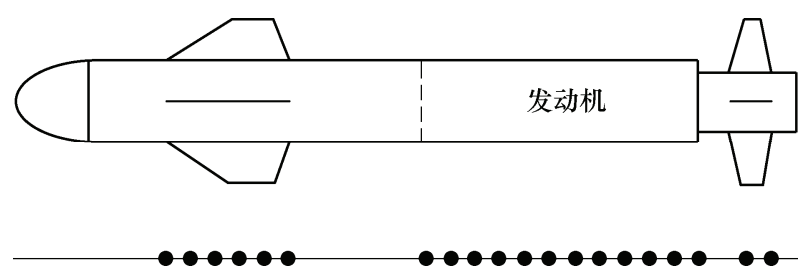

图 2 火箭弹结构与一维梁等效结果模型示意图

在主动段阶段, 发动机上每个节点燃料的质量 可用式(1)表示

$$
m(t)=m_{0}-\Delta m \times t
$$

式中, $t$ 为主动段飞行时间; $m(t)$ 为每个节点上燃料 的质量; $m_{0}$ 为初始时刻每个节点上燃料的质量; $\Delta m$ 为每个节点上的燃料质量秒流量。

远程火箭弹射程远, 飞行时间长, 且火箭弹主 动段不断消耗燃料, 导致火箭弹质量、质心位置、 转动惯量、气动参数、模态振型和刚度矩阵等不断 变化。为加快求解速度, 选取主动段的几个特征点, 用 NASTRAN 求解计算, 得到特征点时刻的模态振 型和刚度矩阵, 然后采用线性插值的方法, 得到主 动段每一时刻的模态振型和刚度矩阵, 同理, 火箭 弹主动段每一时刻的质量、质心位置、转动惯量和 气动参数也通过线性插值的方式求得。

\section{2 远程火箭弹运动建模}

\subsection{1 坐标系的定义}

为了真实准确反映弹性体远程火箭弹空间运 动, 定义发射坐标系 $O x y z$ 和弹体坐标系 $O_{1} x_{1} y_{1} z_{1}$ 。 发射坐标系坐标原点与发射点 $O$ 固连, $O x$ 轴在发 射点水平面内指向发射瞄准方向, $O y$ 轴垂直于发射 点水平面指向上方, $O z$ 轴与 $x O y$ 面相垂直并构成右 手坐标系, 如图 3 所示。弹体坐标系坐标原点 $O_{1}$ 
为火箭弹的质心, $O_{1} x_{1}$ 为火箭弹外壳对称轴, 指向 火箭弹的头部, $O_{1} y_{1}$ 在火箭弹的主对称面内, 该平 面在发射瞬时与发射坐标系 $x O y$ 平面重合, $O y_{1}$ 轴 垂直于 $O x_{1}$ 轴, $O z_{1}$ 轴垂直于主对称面, 在发射瞬时, 顺着发射方向看, $O z_{1}$ 轴指向右方, 如图 4 所示。

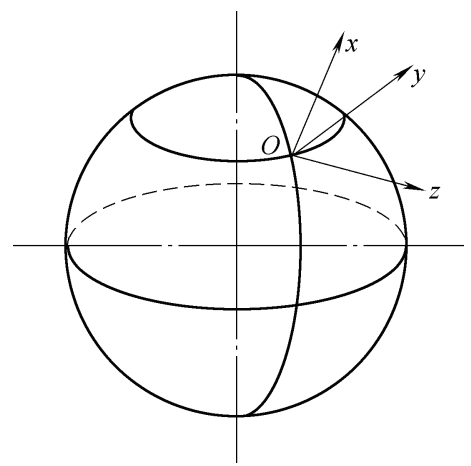

图 3 发射坐标系示意图

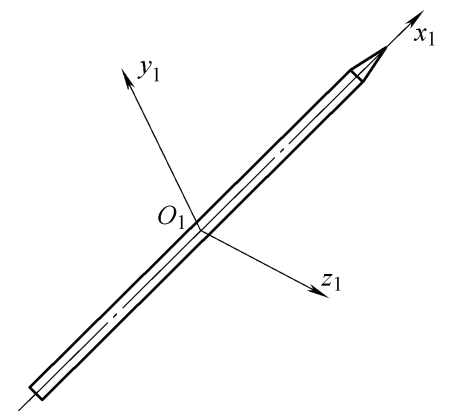

图 4 弹体坐标系示意图

\subsection{2 远程火箭弹气动弹性分析模型}

由于弹性效应，火箭弹在主动段飞行过程中， 喷口处发生弹性变形, 导致推力方向发生改变, 火 箭弹所受的推力和推力矩可用式(2)表示

$$
\left\{\begin{array}{c}
\boldsymbol{F}_{p}=\left(\begin{array}{c}
P \\
P \boldsymbol{\Phi}_{r z}\left(x_{t}\right) \boldsymbol{q} \\
-P \boldsymbol{\Phi}_{r y}\left(x_{t}\right) \boldsymbol{q}
\end{array}\right) \\
\boldsymbol{M}_{p}=\left(\begin{array}{c}
0 \\
P \boldsymbol{\Phi}_{r y}\left(x_{t}\right) \boldsymbol{q} x^{(t)} \\
P \boldsymbol{\Phi}_{r z}\left(x_{t}\right) \boldsymbol{q} x^{(t)}
\end{array}\right)
\end{array}\right.
$$

式中, $\boldsymbol{F}_{p}$ 和 $\boldsymbol{M}_{p}$ 分别表示火箭弹所受的推力和推力 矩; $P$ 为发动机推力; $x^{(t)}$ 为推力作用点坐标; $\boldsymbol{\Phi}_{r z}\left(x_{t}\right)$ 和 $\boldsymbol{\Phi}_{r y}\left(x_{t}\right)$ 分别表示推力作用点沿 $y_{1}$ 轴和 $z_{1}$ 轴方向振 型的转动分量。

此外, 由于气动弹性和结构的耦合作用, 火箭 弹在飞行过程中会受到非定常气动力的影响。非定 常气动力可分别对弹身、弹翼和舵面三部分进行计 算, 其中弹身上的非定常气动力可用细长体理论 ${ }^{[16]}$ 求解, 将火箭弹沿纵向轴分成 $n$ 份, 可得到由各分 段上单位长度上的法向气动力组成矢量

$$
\begin{gathered}
\Delta \boldsymbol{P}=\left[\begin{array}{llll}
\Delta p_{1} & \Delta p_{2} & \cdots & \Delta p_{n}
\end{array}\right]= \\
-\rho\left(\boldsymbol{s} \boldsymbol{\Phi} \ddot{\boldsymbol{q}}+2 V \boldsymbol{s} \boldsymbol{\Phi}^{\prime} \dot{\boldsymbol{q}}+V \boldsymbol{s}^{\prime} \boldsymbol{\Phi} \dot{\boldsymbol{q}}+\right. \\
\left.V^{2} \boldsymbol{s}^{\prime} \boldsymbol{\Phi}^{\prime} \boldsymbol{q}+V^{2} \boldsymbol{s} \boldsymbol{\Phi}^{\prime \prime} \boldsymbol{q}\right)
\end{gathered}
$$

式中, $\Delta \boldsymbol{P}$ 为火箭弹弹身单位长度法向气动力矢量; $\rho$ 为空气密度; $V$ 为来流速度; $\boldsymbol{s}=\operatorname{diag}\left(s_{1}, s_{2}, \cdots, s_{n}\right)$ 为火箭弹各横截面积组成的对角矩阵。

运用细长体理论, 弹身产生的非定常气动力和 力矩的计算公式如式(4)所示

$$
\left\{\begin{array}{l}
F_{\text {unst_body }}=\boldsymbol{E}(\boldsymbol{L} \Delta \boldsymbol{P}) \\
M_{\text {unst_body }}=\boldsymbol{X}(\boldsymbol{L} \Delta \boldsymbol{P})
\end{array}\right.
$$

式中, $\boldsymbol{E}=\left(\begin{array}{llll}1 & 1 & \cdots & 1\end{array}\right)$ 为一个 $1 \times n$ 的向量, 其中每个 元素均为 $1 ; \boldsymbol{X}=\left(x_{1} x_{2} \cdots x_{n}\right)$ 为火箭弹弹身各段中 点坐标组成的矢量; $\boldsymbol{L}=\left(l_{1} l_{2} \cdots l_{n}\right)$ 为火箭弹弹身 各段长度组成的矢量。

对于弹翼和舵面, 采用气动导数法 ${ }^{[16]}$ 计算弹性 变形产生的附加攻角、侧滑角和舵偏角, 如式(5) 所示

$$
\left\{\begin{array}{l}
u_{y}\left(x_{f}\right)=\boldsymbol{\Phi}_{t y}\left(x_{f}\right) \boldsymbol{q}, u_{z}\left(x_{f}\right)=\boldsymbol{\Phi}_{t z}\left(x_{f}\right) \boldsymbol{q} \\
u_{y}\left(x_{r}\right)=\boldsymbol{\Phi}_{t y}\left(x_{r}\right) \boldsymbol{q}, u_{z}\left(x_{r}\right)=\boldsymbol{\Phi}_{t z}\left(x_{r}\right) \boldsymbol{q} \\
\alpha_{\text {add }}=\frac{\partial u_{y}\left(x_{f}\right)}{\partial x}-\frac{\dot{u}_{y}\left(x_{f}\right)}{V} \\
\beta_{\text {add }}=-\frac{\partial u_{z}\left(x_{f}\right)}{\partial x}+\frac{\dot{u}_{z}\left(x_{f}\right)}{V} \\
\delta_{\text {zadd }}=\frac{\partial u_{y}\left(x_{r}\right)}{\partial x} \\
\delta_{\text {yadd }}=-\frac{\partial u_{z}\left(x_{r}\right)}{\partial x}
\end{array}\right.
$$

式中, $u_{y}\left(x_{f}\right)$ 和 $u_{z}\left(x_{f}\right)$ 分别表示火箭弹弹翼位置沿 $y$ 轴和 $z$ 轴方向的变形量; $u_{y}\left(x_{r}\right)$ 和 $u_{z}\left(x_{r}\right)$ 分别表示火 箭弹舵面位置沿 $y_{1}$ 轴和 $z_{1}$ 轴方向的变形量; $\boldsymbol{\Phi}_{t y}\left(x_{f}\right)$ 和 $\boldsymbol{\Phi}_{t z}\left(x_{f}\right)$ 分别表示火箭弹弹翼位置沿 $y_{1}$ 轴和 $z_{1}$ 轴方向 振型的平动分量; $\boldsymbol{\Phi}_{t y}\left(x_{r}\right)$ 和 $\boldsymbol{\Phi}_{t z}\left(x_{r}\right)$ 分别表示火箭弹 舵面位置沿 $y_{1}$ 轴和 $z_{1}$ 轴方向振型的平动分量; $\alpha_{a d d}$ 和 $\beta_{\text {add }}$ 分别表示附加攻角和附加侧滑角; $\delta_{z a d d}$ 和 $\delta_{\text {yadd }}$ 分 别表示附加俯仰舵偏角和附加偏航舵偏角。

由弹翼和舵面产生的非定常气动力和力矩可用 式(6)、(7)表示

$$
\left\{\begin{aligned}
& \boldsymbol{F}_{\text {unst_fin }}=\left(\begin{array}{c}
0 \\
c_{N}^{\alpha}\left(x_{f}\right) \alpha_{\text {add }} \\
c_{Z}^{\beta}\left(x_{f}\right) \beta_{\text {add }}
\end{array}\right) Q S \\
& 0 \\
& \boldsymbol{M}_{\text {unst_fin }}=\left(\begin{array}{c}
0 \\
-c_{Z}^{\beta}\left(x_{f}\right) \beta_{\text {add }} \\
c_{N}^{\alpha}\left(x_{f}\right) \alpha_{\text {add }}
\end{array}\right) Q S x^{(f)}
\end{aligned}\right.
$$




$$
\left\{\begin{array}{l}
\boldsymbol{F}_{\text {unst_rud }}=\left(\begin{array}{c}
0 \\
c_{N}^{\delta_{z}}\left(x_{r}\right) \delta_{\text {zadd }} \\
c_{Z}^{\delta_{y}}\left(x_{r}\right) \delta_{\text {yadd }}
\end{array}\right) Q S \\
\boldsymbol{M}_{\text {unst_rud }}=\left(\begin{array}{c}
0 \\
-c_{Z}^{\delta_{y}}\left(x_{r}\right) \delta_{\text {yadd }} \\
c_{N}^{\delta_{z}}\left(x_{r}\right) \delta_{\text {zadd }}
\end{array}\right) Q S x^{(r)}
\end{array}\right.
$$

式中, $c_{N}^{\alpha}\left(x_{f}\right)$ 和 $c_{Z}^{\beta}\left(x_{f}\right)$ 分别表示单独弹翼的法向力 系数关于攻角的导数和侧向力系数关于侧滑角的导 数; $c_{N}^{\delta_{z}}\left(x_{r}\right)$ 和 $c_{Z}^{\delta_{y}}\left(x_{r}\right)$ 分别表示单独舵面的法向力系 数关于俯仰舵偏角的导数和侧向力系数关于偏航 舵偏角的导数; $x^{(f)}$ 和 $x^{(r)}$ 分别表示弹翼和舵面的 坐标。

\subsection{3 远程火箭弹动力学模型}

将火箭弹空间运动视作具有六个自由度的可控 制的变质量系统运动, 可得火箭弹质心运动、绕质 心转动和弹性振动的三个矢量方程如下 ${ }^{[12,17-19]}$

$$
\left\{\begin{array}{l}
M \dot{\boldsymbol{v}}=\boldsymbol{F}_{\text {all }} \\
\boldsymbol{J} \dot{\boldsymbol{\omega}}=\boldsymbol{M}_{\text {all }}-\boldsymbol{\omega} \times(\boldsymbol{J} \boldsymbol{\omega}) \\
\boldsymbol{M}_{q} \ddot{\boldsymbol{q}}+\boldsymbol{C}_{q} \dot{\boldsymbol{q}}+\boldsymbol{K}_{q} \boldsymbol{q}=\boldsymbol{\Phi}_{t}^{T} \boldsymbol{Q}_{a l l}
\end{array}\right.
$$

式中, $M$ 为火箭弹质量; $\boldsymbol{J}$ 为火箭弹转动惯量矩阵; $v$ 为火箭弹在发射坐标系下的质心运动速度, $\boldsymbol{v}=\left(\begin{array}{lll}v_{x} & v_{y} & v_{z}\end{array}\right)^{\mathrm{T}} ; \boldsymbol{\omega}$ 为火箭弹在弹体坐标系下的转动 角速度, $\boldsymbol{\omega}=\left(\omega_{x} \omega_{y} \omega_{z}\right)^{\mathrm{T}} ; \boldsymbol{q}$ 为弹性振动的模态坐 标, $\boldsymbol{q}=\left(q_{z 1} q_{z 2} q_{z 3} q_{y 1} q_{y 2} q_{y 3}\right)^{\mathrm{T}}$, 其中 $q_{z 1} 、 q_{z 2}$ 和 $q_{z 3}$ 分别表示火箭弹纵向前三阶弹性振动的模态坐标, $q_{y 1} 、 q_{y 2}$ 和 $q_{y 3}$ 分别表示火箭弹侧向前三阶弹性振动 的模态坐标; $\boldsymbol{\Phi}_{t}$ 为弹体模态振型的平动分量; $\boldsymbol{F}_{\text {all }}$ 为火箭弹所受到的合外力; $\boldsymbol{M}_{\text {all }}$ 为火箭弹所受到的 合外力矩; $\boldsymbol{Q}_{\text {all }}$ 为沿火箭弹弹轴方向的分布力矩阵; $\boldsymbol{M}_{q} 、 \boldsymbol{C}_{q}$ 和 $\boldsymbol{K}_{q}$ 分别表示火箭弹结构质量矩阵、阻尼 矩阵和刚度矩阵。

作用在火箭弹上的外力有地球引力、推力、附 加哥氏力、离心惯性力、哥氏惯性力和空气动力 ${ }^{[20]}$ 。

(1) 地球引力。地球引力可用式(9)、(10)和(11) 表示

$$
\begin{gathered}
M \boldsymbol{g}=M g_{r}^{\prime} \boldsymbol{r}^{0}+M g_{\omega e} \boldsymbol{\omega}_{e}^{0} \\
g_{r}^{\prime}=-\frac{f M_{1}}{r^{2}}\left[1+J\left(\frac{a_{e}}{r}\right)^{2}\left(1-5 \sin ^{2} \phi\right)\right] \\
g_{\omega e}=-2 \frac{f M_{1}}{r^{2}} J\left(\frac{a_{e}}{r}\right)^{2} \sin \phi
\end{gathered}
$$

式中, $\boldsymbol{g}$ 为引力加速度矢量; $g_{r}^{\prime}$ 为引力加速度矢量 在子午面内的分量; $g_{\omega e}$ 为引力加速度矢量垂直于
子午面的分量; $\boldsymbol{r}^{0}$ 为火箭弹与地心连线在子午面内 投影的单位矢量; $\omega_{e}^{0}$ 为火箭弹与地心连线垂直于子 午面的单位矢量; $f$ 为万有引力常数; $M_{1}$ 为地球质 量; $r$ 为火箭弹距地心的距离; $J$ 为带谐系数; $a_{e}$ 为 地球赤道平均半径; $\phi$ 为地心纬度。

(2) 推力。考虑到火箭弹喷口处的弹性变形, 火箭弹所受的推力和推力矩可用式(2)表示。

(3) 附加哥式力。附加哥氏力是由于火箭弹主 动段阶段燃料的消耗, 导致质心位置的变化, 从而 产生的一个相对力。火箭弹所受的附加哥氏力可用 式(12)表示

$$
\boldsymbol{F}_{k}^{\prime}=-2 \dot{M} \boldsymbol{\omega}_{T} \times \boldsymbol{\rho}_{e}
$$

式中, $\boldsymbol{F}_{k}^{\prime}$ 为火箭弹所受的附加哥氏力; $\dot{M}$ 为燃料 消耗的秒流量; $\omega_{T}$ 为火箭弹相对于惯性坐标系的转 动角速度矢量; $\rho_{e}$ 为质心到喷管出口中心点的矢量。

(4) 离心惯性力和哥氏惯性力。由于地球自转, 火箭弹受到离心惯性力和哥氏惯性力的作用, 其表 达式如式(13)和式(14)所示

$$
\begin{gathered}
\boldsymbol{F}_{e}=-M \boldsymbol{\omega}_{e} \times\left(\boldsymbol{\omega}_{e} \times \boldsymbol{r}\right) \\
\boldsymbol{F}_{k}=-2 M \boldsymbol{\omega}_{e} \times \frac{\delta \boldsymbol{r}}{\delta t}
\end{gathered}
$$

式中, $\boldsymbol{F}_{e}$ 为火箭弹所受的离心惯性力; $M$ 为火箭弹 总质量; $\omega_{e}$ 为地球自转角速度矢量; $\boldsymbol{r}$ 为火箭弹与 地心之间的矢径; $\boldsymbol{F}_{k}$ 为火箭弹所受的哥氏惯性力; $\frac{\delta \boldsymbol{r}}{\delta t}$ 为火箭弹相对于发射坐标系的速度。

(5) 空气动力。火箭弹受到的空气动力可分为 定常气动力和非定常气动力, 其中, 定常气动力和 力矩可用式(15)表示 ${ }^{[21]}$

$$
\left\{\begin{array}{l}
\boldsymbol{F}_{s t}=\left(\begin{array}{lll}
-c_{A} & c_{N} & c_{Z}
\end{array}\right)^{\mathrm{T}} Q S \\
\boldsymbol{M}_{s t}=\left(\begin{array}{lll}
-m_{x} & m_{y} & m_{z}
\end{array}\right)^{\mathrm{T}} Q S l
\end{array}\right.
$$

式中, $\boldsymbol{F}_{s t}$ 和 $\boldsymbol{M}_{s t}$ 分别为定常气动力和定常气动力 矩; $Q$ 为动压; $S$ 为火箭弹特征面积; $l$ 为火箭弹特 征长度; $c_{A} 、 c_{N}$ 和 $c_{Z}$ 分别表示火箭弹轴向力、法向 力和侧向力系数; $m_{x} 、 m_{y}$ 和 $m_{z}$ 分别表示火箭弹滚 转力矩系数、偏航力矩系数和俯仰力矩系数。

弹身、弹翼和舵面产生的非定常气动力和力矩 如式(4)、式(6)和式(7)所示。

\section{3 远程火箭弹制导控制方案}

为了实现火箭弹在现代战争中精确打击的要 求, 通常采用三回路自动驾驶仪来提高控制精度。

远程火箭弹从炮管打出后, 纵向先进行等弹道 倾角爬升, 中间过程保持无控飞行，末制导采用带 落角约束的比例导引制导律 ${ }^{[22]}$ 。忽略舵机动力学环 
节, 俯仰方向舵偏角变化规律如式(16)所示, 纵向等弹道倾角爬升和末制导指令信号如式(17)所示

$$
\begin{aligned}
& \delta_{z}= \begin{cases}k_{A C T} K_{D C} K_{A} V \theta^{*}+k_{A C T} k_{a c} K_{A} V \alpha \\
-k_{A C T}\left(k_{a c} K_{A} V+\omega_{I}\right) \vartheta-k_{A C T}\left(k_{g}+c k_{a c} K_{A}\right) \dot{\vartheta} & 0<t<t_{0} \text { 或 } r<r_{0} \\
0 & t \geqslant t_{0} \text { 且 } r \geqslant r_{0}\end{cases} \\
& \theta^{*}= \begin{cases}\theta_{0} & 0<t<t_{0} \\
\left(N_{P} V \dot{q}_{z}+\frac{N_{\theta} V\left(q_{z}-q_{F}\right)}{t_{g o}}+N_{a_{T}} g \cos \theta\right) / V+\theta & r<r_{0}\end{cases} \\
& \delta_{y}= \begin{cases}k_{A C T} K_{D C} K_{A} V \varphi_{v}^{*}+k_{A C T} k_{a c} K_{A} V \beta \\
-k_{A C T}\left(k_{a c} K_{A} V+\omega_{I}\right) \varphi-k_{A C T}\left(k_{g}+c k_{a c} K_{A}\right) \dot{\varphi} & r<r_{0} \\
0 & r \geqslant r_{0}\end{cases} \\
& \varphi_{v}^{*}=N \dot{q}_{c}+\varphi_{v}
\end{aligned}
$$

式中, $\delta_{z}$ 为俯仰方向舵偏角; $k_{A C T}$ 为舵机增益; $k_{a c}$ 为加速度计增益; $K_{D C} 、 K_{A} 、 \omega_{I}$ 以及 $k_{g}$ 为自驾仪设 计参数; $c$ 为加速度计放置位置; $t_{0}$ 为主动段结束时 间; $r_{0}$ 为进入末制导时的弹目距离; $V$ 为火箭弹的 速度; $\alpha$ 为攻角; $\vartheta$ 为俯仰角; $\dot{\vartheta}$ 为俯仰角速度; $\theta^{*}$ 为纵向控制指令; $\theta_{0}$ 为火箭弹爬升段弹道倾角信 号; $N_{P} 、 N_{\vartheta} 、 N_{a_{T}}$ 分别表示位置项、落角项、目标 机动项的导航系数; $q_{z}$ 为弹目视线角垂直分量; $\dot{q}_{z}$ 为 弹目视线角速度垂直分量; $q_{F}$ 为落角约束; $\theta$ 为火 箭弹弹道倾角; $t_{g o}$ 为火箭弹此刻到击中目标的时间。

火箭弹侧向采用比例导引制导律, 侧向偏航方 向舵偏角变化规律如式(18)所示, 侧向末制导指令 信号如式(19)所示。其中, $\delta_{y}$ 为偏航方向舵偏角; $\beta$ 为侧滑角; $\varphi$ 为偏航角; $\dot{\varphi}$ 为偏航角速度; $\varphi_{v}^{*}$ 为侧 向控制指令; $N$ 为侧向比例导引系数; $\dot{q}_{c}$ 为弹目视 线角速度水平分量; $\varphi_{v}$ 为弹道偏角。

考虑到火箭弹上的敏感器件(陀螺仪和加速度 计)固连在火箭弹弹轴上, 由于弹性振动的影响, 会 使测得的法向加速度和角速率失准, 实际测得值如 式(20)所示

$$
\left\{\begin{array}{l}
n_{y}^{*}=\dot{v}_{y}+x^{(a)} \dot{\omega}_{z}+\boldsymbol{\Phi}_{t y}\left(x_{a}\right) \ddot{\boldsymbol{q}} \\
n_{z}^{*}=\dot{v}_{z}-x^{(a)} \dot{\omega}_{y}+\boldsymbol{\Phi}_{t z}\left(x_{a}\right) \ddot{\boldsymbol{q}} \\
\omega_{y}^{*}=\omega_{y}-\boldsymbol{\Phi}_{r y}\left(x_{g}\right) \dot{\boldsymbol{q}} \\
\omega_{z}^{*}=\omega_{z}+\boldsymbol{\Phi}_{r z}\left(x_{g}\right) \dot{\boldsymbol{q}}
\end{array}\right.
$$

式中, $n_{y}^{*}$ 和 $n_{z}^{*}$ 分别表示实际测得的火箭弹沿弹体 $y_{1}$ 轴和 $z_{1}$ 轴的法向过载; $\omega_{y}^{*}$ 和 $\omega_{z}^{*}$ 分别表示实际测 得的火箭弹绕弹体 $y_{1}$ 轴和 $z_{1}$ 轴的角速度; $\dot{v}_{y}$ 和 $\dot{v}_{z}$ 分 别表示火箭弹沿弹体 $y_{1}$ 轴和 $z_{1}$ 轴速度的导数; $\omega_{y}$ 和 $\omega_{z}$ 分别表示火箭弹绕弹体 $y$ 轴和 $z$ 轴的角速度; $x^{(a)}$ 为火箭弹加速度计安装位置的坐标; $\boldsymbol{\Phi}_{t y}\left(x_{a}\right)$ 和 $\boldsymbol{\Phi}_{t z}\left(x_{a}\right)$ 分别表示火箭弹加速度计位置沿 $y_{1}$ 轴和 $z_{1}$ 轴
方向振型的平动分量; $\boldsymbol{\Phi}_{r y}\left(x_{g}\right)$ 和 $\boldsymbol{\Phi}_{r z}\left(x_{g}\right)$ 分别表示火箭 弹陀螺仪位置沿 $y_{1}$ 轴和 $z_{1}$ 轴方向振型的转动分量。

\section{2 仿真试验与分析}

在弹性体弹箭弹道研究方面, 文献[10-11]指 出考虑气动弹性有利于提高弹箭的弹道仿真精 度。然而, 气动弹性与附加力等因素对弹箭飞行 性能及脱靶量的影响研究较为少见。为了进一步 揭示影响弹性体远程火箭弹弹道仿真结果的因 素, 本节以第 1 节中的飞行仿真模型为基础, 分 别对气动弹性、附加力和结构刚度对脱靶量的影 响进行了详细分析。

\section{1 仿真设置}

为了分别比较气动弹性、附加力和结构刚度对 火箭弹脱靶量的影响, 以某型火箭弹为例, 进行六 自由度弹道仿真, 火箭弹基本参数如表 1 所示, 一 维梁结构图如图 5 所示。

表 1 火箭弹基本参数定义

\begin{tabular}{lc||lc}
\hline \multicolumn{1}{c|}{ 参数 } & 取值 & \multicolumn{1}{|c}{ 参数 } & 取值 \\
\hline 主动段/被动段质量 $/ \mathrm{kg}$ & 1290 & 弹翼展长 $/ \mathrm{m}$ & 0.18 \\
& 560 & 弹翼前缘后掠角 $/\left(^{\circ}\right)$ & 40 \\
主动段时间 $/ \mathrm{s}$ & $0 \sim 22$ & NACA \\
火箭弹总长 $/ \mathrm{m}$ & 8 & 弹翼截面形状 & 0012 \\
弹头长度 $/ \mathrm{m}$ & 0.32 & 舵面根弦长 $/ \mathrm{m}$ & 0.31 \\
弹身直径 $/ \mathrm{m}$ & 0.29 & 舵面梢弦长 $/ \mathrm{m}$ & 0.13 \\
尾部长度 $/ \mathrm{m}$ & 0.71 & 舵面展长 $/ \mathrm{m}$ & 0.13 \\
弹翼根弦长 $/ \mathrm{m}$ & 0.61 & 舵面前缘后掠角 $\left./{ }^{\circ}\right)$ & 50 \\
弹翼梢弦长 $/ \mathrm{m}$ & & 舵面截面形状 & $\mathrm{NACA}$ \\
\hline
\end{tabular}

图 5 火箭弹一维梁结构图 
对于算例中的火箭弹, 被动段前三阶模态频率 分别为 $16 \mathrm{~Hz} 、 42 \mathrm{~Hz}$ 和 $77 \mathrm{~Hz}$, 其模态振型的平动 分量如图 6 所示。火箭弹弹道设计技术指标和发射 参数分别如表 2 和表 3 所示, 目标位置示意图如图 7 所示。

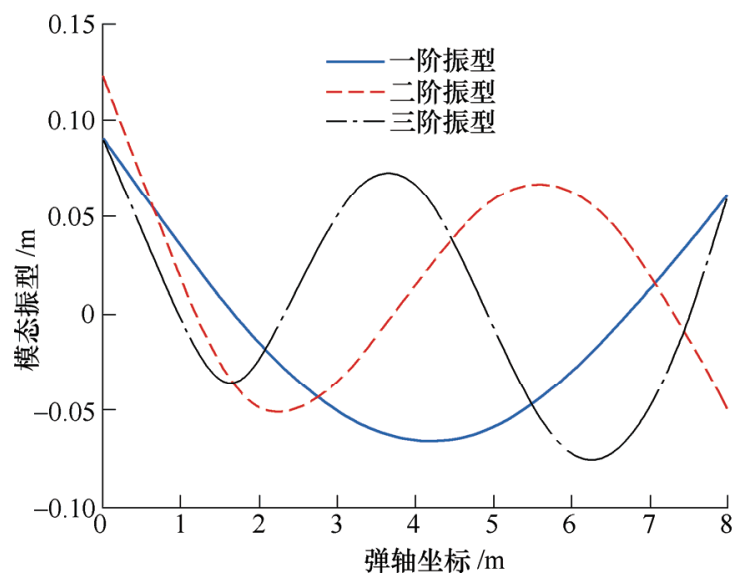

图 6 火箭弹被动段前三阶模态振型平动分量

表 2 弹道设计技术指标

\begin{tabular}{ccccc}
\hline 参数 & 期望落角 $/\left(^{\circ}\right)$ & 脱靶量 $/ \mathrm{m}$ & $\begin{array}{c}\text { 舵偏角 } \\
\text { 变化范围 } /\left(^{\circ}\right)\end{array}$ & 射程 $/ \mathrm{km}$ \\
\hline 取值 & 80 & $\leqslant 10$ & $-15 \sim 15$ & 180 \\
\hline
\end{tabular}

表 3 火箭弹发射参数

\begin{tabular}{cccccc}
\hline 参数 & 经度 $/\left(^{\circ}\right)$ & 纬度 $/\left(^{\circ}\right)$ & 海拔 $/ \mathrm{km}$ & 方向 & 推力 $/ \mathrm{kN}$ \\
\hline 取值 & 东经 90 & 北纬 30 & 1.4 & 北 & $0 \sim 5 \mathrm{~s}: 140$ \\
& & & & & $5 \sim 22 \mathrm{~s}: 70$ \\
\hline
\end{tabular}

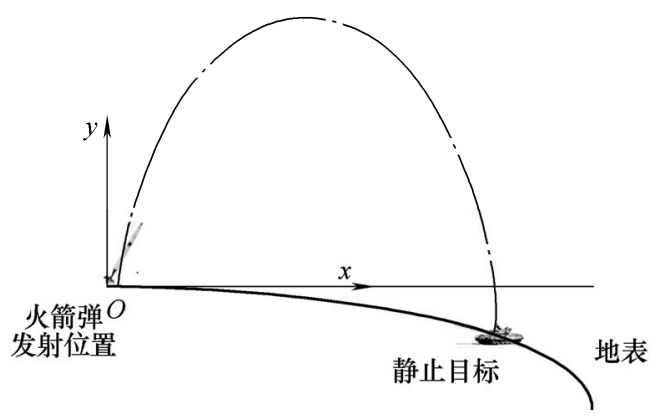

图 7 目标位置示意图

\section{2 气动弹性和附加力影响对比}

在仿真试验中, 火箭弹以零初速从 $8 \mathrm{~m}$ 长的炮 管中发射, 火箭弹在炮管内只考虑重力和推力的作 用, 如图 8 所示。

以考虑附加力刚体火箭弹的弹道模型为基准, 保持控制参数不变, 可分别得到考虑附加力弹性体、 忽略附加力刚体和忽略附加力弹性体火箭弹的弹道 模型。通过弹道仿真, 得到射高、侧偏、弹道倾角、 舵偏角、法向过载和弹头变形曲线如图 9 14 所示, 火箭弹脱靶量对比如表 4 所示。

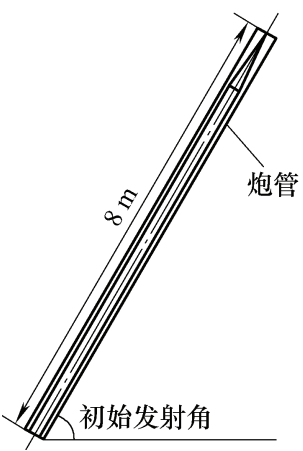

图 8 火箭弹的发射示意图

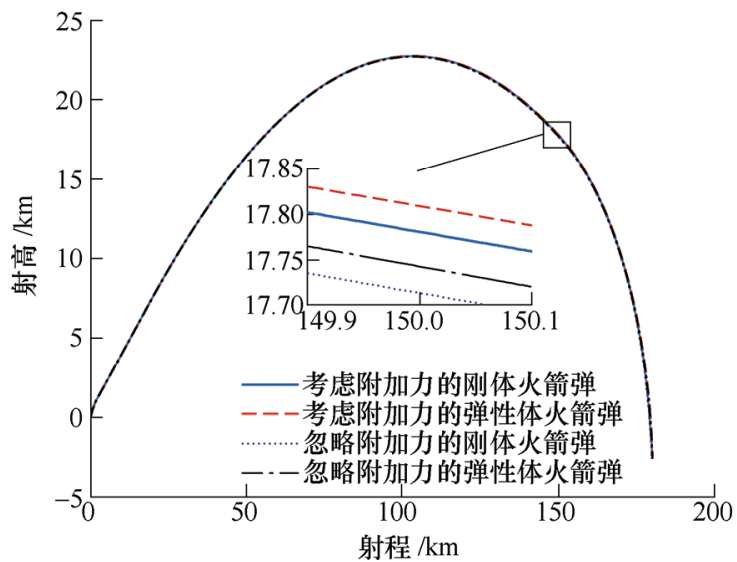

图 9 射高曲线

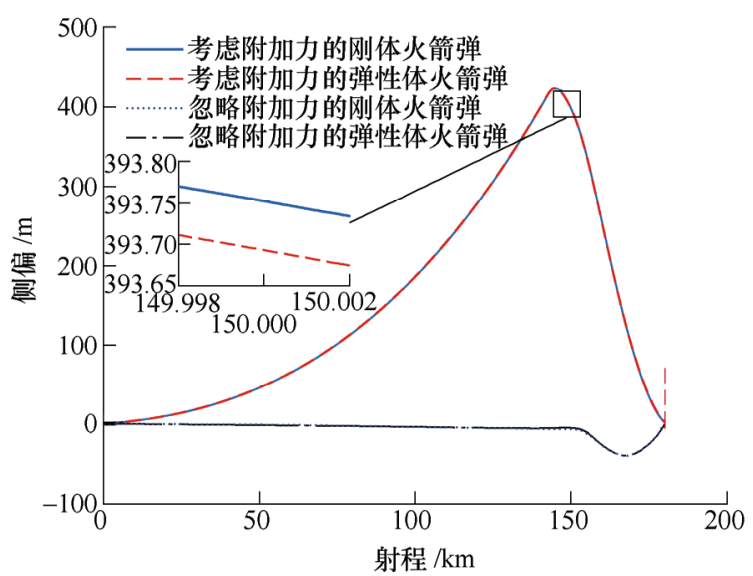

图 10 侧偏曲线

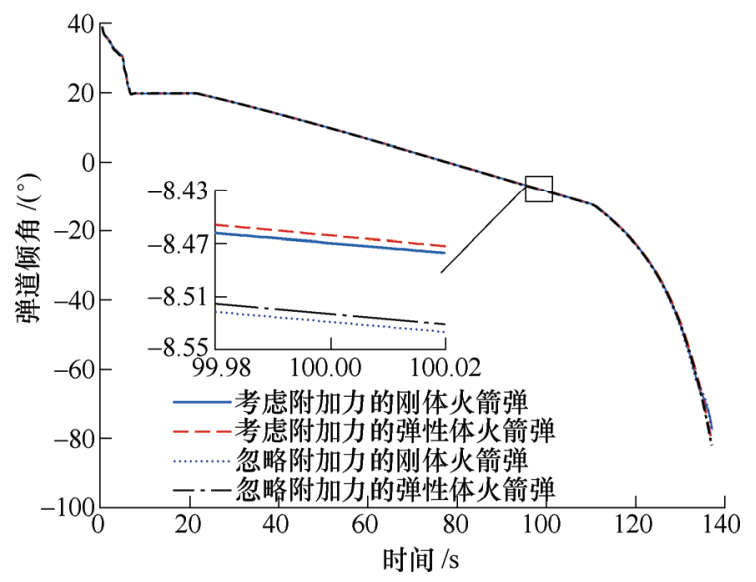

图 11 弹道倾角曲线 
从图 9 和图 10 可以看出, 考虑附加力刚体、考 虑附加力弹性体、忽略附加力刚体和忽略附加力弹 性体火箭弹的射高和侧偏曲线有明显差异, 可以看 出火箭弹弹性变形产生的弹性效应以及地球自转和 燃料变化产生的附加力的影响。

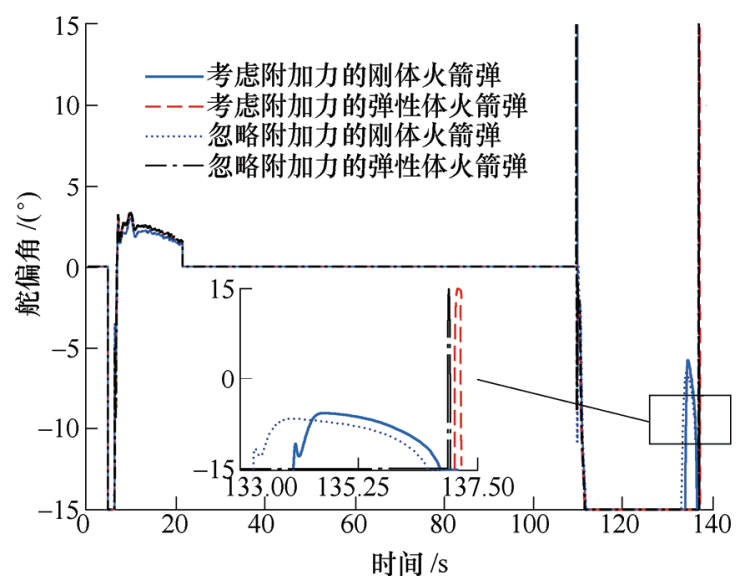

图 12 舵偏角曲线

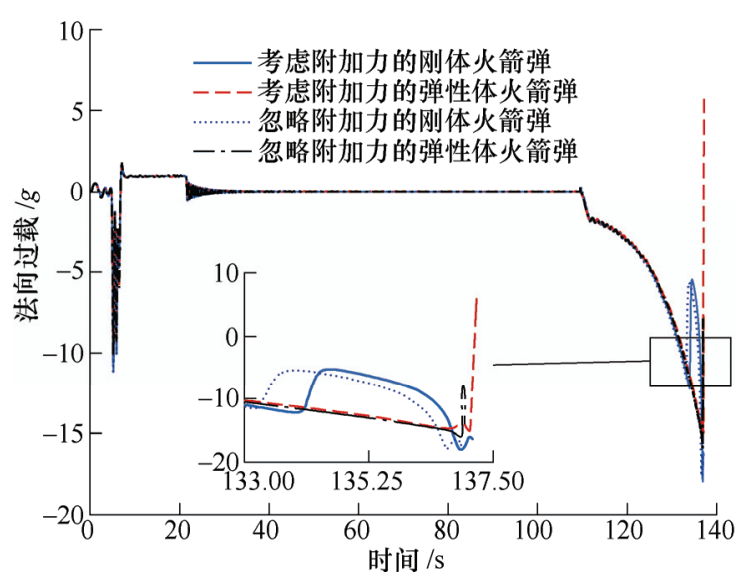

图 13 法向过载曲线

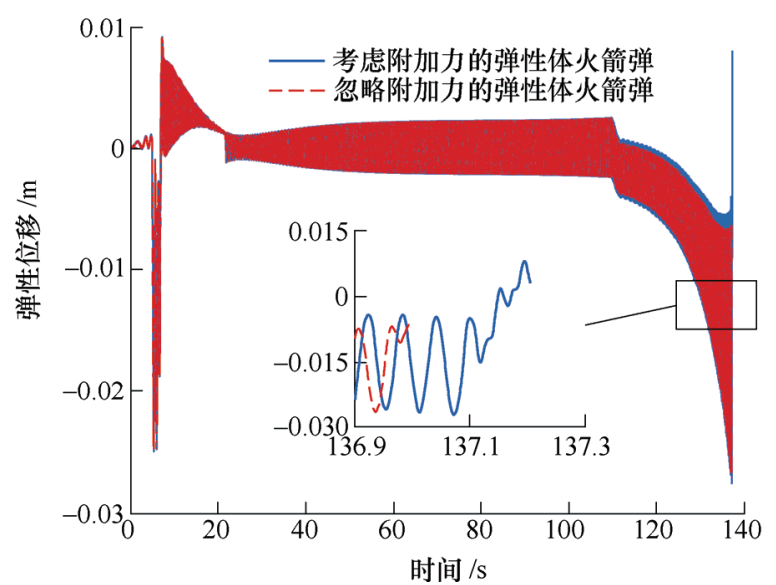

图 14 弹头变形曲线

从图 11 可以看出, 火箭弹在 $0 \sim 22 \mathrm{~s}$ 内进行 等弹道倾角爬升, $110 \mathrm{~s}$ 之后进入带落角约束的比 例导引阶段。从图 12 和图 13 可以看出, 火箭弹 法向过载在 $5 \mathrm{~s}$ 左右发生振荡, 因为在此时刻推力
发生变化, 导致舵偏角发生突变，从而激发了弹 体运动。

图 12 中，火箭弹进入末制导阶段之后，由于 弹性效应, 考虑附加力弹性体和忽略附加力弹性 体火箭弹的舵偏角分别在 $136.9 \mathrm{~s}$ 和 $137.1 \mathrm{~s}$ 之前一 直处于满偏状态，且考虑附加力弹性体和忽略附 加力弹性体火箭弹分别在 $136.9 \mathrm{~s}$ 和 $137.1 \mathrm{~s}$ 时达到 射程要求，但两者都仍然处于飞行状态，所以下 一时刻弹目视线角会发生突变，从而导致舵偏角 发生突变。

图 13 中, 考虑附加力弹性体和忽略附加力弹性 体火箭弹的法向过载分别在 $136.9 \mathrm{~s}$ 和 $137.1 \mathrm{~s}$ 时发 生突变, 且法向过载的最大变化量可分别达到 $8 \mathrm{~g}$ 和 $21 \mathrm{~g}$ 。由于法向过载的突变, 引起弹体形变量发 生变化 (以弹头为例, 对整个弹体的形变量进行分析) 如图 14 所示, 分别在 $136.9 \mathrm{~s}$ 和 $137.1 \mathrm{~s}$ 之后, 考虑 附加力弹性体和忽略附加力弹性体火箭弹弹头变形 量的最大变化量可分别达到 $4.5 \mathrm{~mm}$ 和 $22 \mathrm{~mm}$ 。

表 4 考虑不同因素影响的火箭弹脱靶量对比

\begin{tabular}{cc}
\hline 考虑不同因素影响的弹道模型 & 脱靶量 $/ \mathrm{m}$ \\
\hline 考虑附加力的弹性体火箭弹 & 158.1 \\
忽略附加力的弹性体火箭弹 & 44.7 \\
考虑附加力的刚体火箭弹 & 3.7 \\
忽略附加力的刚体火箭弹 & 3.4 \\
\hline
\end{tabular}

对比表 4 中的数据可知, 考虑附加力弹性体火 箭弹的脱靶量大于考虑附加力刚体火箭弹的脱靶 量, 忽略附加力弹性体火箭弹的脱靶量大于忽略附 加力刚体火箭弹的脱靶量。因此, 气动弹性效应会 导致火箭弹脱靶量增加。更重要的是，与忽略附加 力的弹性体火箭弹相比, 考虑附加力的弹性体火箭 弹脱靶量增加了 $253.7 \%$; 而考虑附加力的刚体火箭 弹脱靶量仅比忽略附加力的刚体火箭增加了 $8.1 \%$ 。 上述对比数据表明: 远程火箭弹弹道仿真中附加力 与气动弹性高度耦合, 在考虑气动弹性效应的前提 下，附加力对火箭弹脱靶量的影响被显著放大。因 此, 为了真实地反映火箭弹的命中精度, 必须综合 考虑火箭弹的气动弹性效应以及地球自转和燃料变 化产生的附加力等多种因素。

\section{3 结构刚度影响对比}

考虑气动弹性、地球自转和燃料变化产生的附 加力影响, 调整火箭弹结构刚度, 使其被动段基频 分别为 $13 \mathrm{~Hz} 、 16 \mathrm{~Hz} 、 19 \mathrm{~Hz}$ 和 $23 \mathrm{~Hz}$ 。通过弹道仿 真，可得到不同结构刚度条件下弹性体火箭弹的射 高和侧偏曲线分别如图 15 和图 16 所示，非定常气 动力对比曲线如图 17 所示, 脱靶量对比如表 5 所示。 


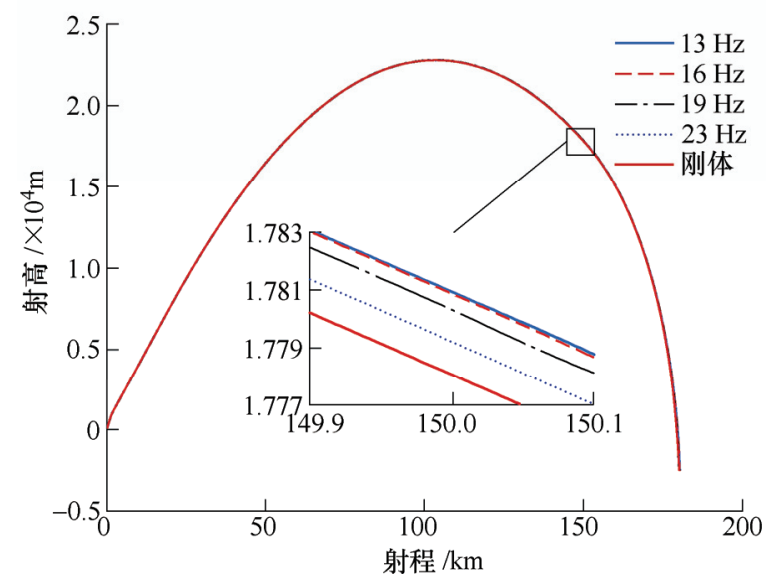

图 15 不同结构刚度火箭弹的射高曲线

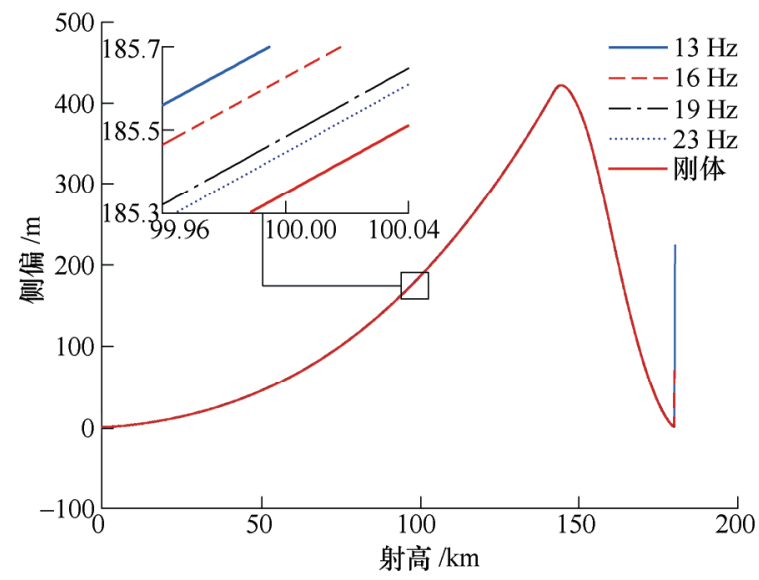

图 16 不同结构刚度火箭弹的侧偏曲线

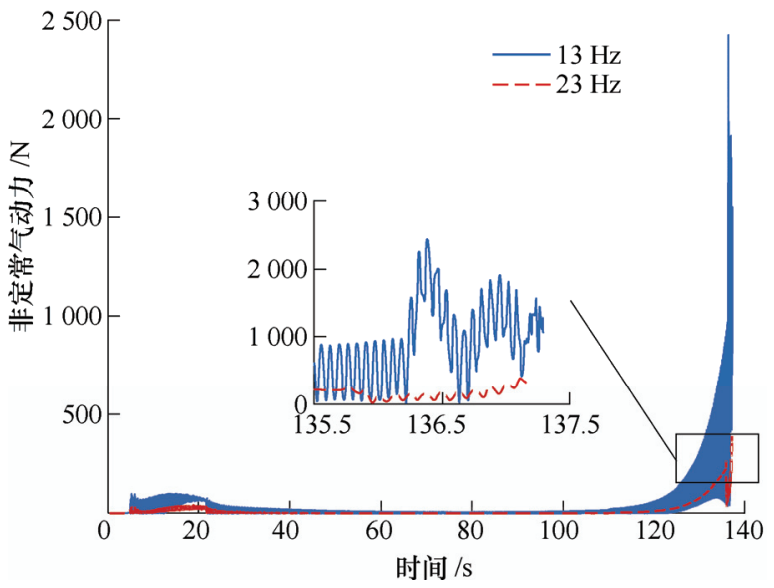

图 17 低刚度和高刚度火箭弹非定常气动力曲线

表 5 不同结构刚度火箭弹的脱靶量

\begin{tabular}{cccccc}
\hline 弹体基频 $/ \mathrm{Hz}$ & 13 & 16 & 19 & 23 & 刚体 \\
\hline 脱靶量 $/ \mathrm{m}$ & 377.8 & 158.1 & 13.9 & 9.6 & 3.7 \\
\hline
\end{tabular}

从图 15 和图 16 可以看出, 随着弹体结构刚度 的提高, 弹性体火箭弹的射高和侧偏曲线与刚体火 箭弹越来越接近。从表 5 和图 17 可以看出, 当火箭 弹的弹体基频为 $13 \mathrm{~Hz}$ 时, 由于结构弹性变形而产 生的非定常气动力较大, 导致其脱靶量为 $377.8 \mathrm{~m}$; 而当火箭弹的弹体基频为 $23 \mathrm{~Hz}$ 时, 结构弹性变形
而产生的非定常气动力较小, 因此其脱靶量仅为 9.6 $\mathrm{m}$ 且与刚体火箭弹的脱靶量十分接近。由此可以说 明, 当其他条件保持不变时, 随着结构刚度的减小, 火箭弹的脱靶量增大, 同时也说明了弹性体远程火 箭弹的飞行仿真建模是正确合理的。

上述结果表明: 考虑气动弹性有利于提高远程 火箭弹的弹道仿真精度, 地球自转和发动机燃料变 化产生的附加力对弹性体远程火箭弹弹道仿真脱靶 量具有较大影响。为了保证设计质量与可信度, 在 远程火箭弹高精度弹道仿真有必要充分考虑上述因 素的影响。

\section{3 结论}

为了进一步揭示影响弹性体远程火箭弹弹道仿 真的因素, 本文建立了一套综合考虑气动弹性、地 球自转和发动机燃料变化产生的附加力等影响的全 弹道分析模型, 通过仿真, 分别对比分析了气动弹 性、附加力和结构刚度对脱靶量的影响, 得到结论 如下。

（1）对于弹性体远程火箭弹, 由于刚体运动和 弹性振动相互耦合, 弹性变形产生的非定常气动力 会导致火箭弹脱靶量增大, 此外, 地球自转和发动 机燃料变化产生的附加力对火箭弹脱靶量有较大影 响。因此, 在高精度弹道仿真中, 有必要同时考虑 气动弹性和附加力的影响。

(2) 地球自转和发动机燃料变化产生的附加力 与气动弹性高度耦合, 在考虑气动弹性效应的前提 下, 附加力对火箭弹脱靶量的影响被显著放大。

（3）仿真试验结果表明弹体结构刚度越小, 弹性 体远程火箭弹脱靶量越大。因此, 在火箭弹的初步 设计中, 应保证火箭弹的结构刚度以确保命中精度。

\section{参 考 文 献}

[1] 邢立新, 吴新明, 㚞海刚. 远程火箭弹任务分析 [J]. 弹 箭与制导学报，2007，27(3): 258-260.

XING Lixin, WU Xinming, FAN Haigang. Long range rocket projectile task analysis[J]. Journal of Projectile,

Rockets, Missile and Guidance, 2007, 27(3): 258-260.

[2] ZHAO L Y, PARK G J, LEE K S, et al. Conceptual design of wrap-around-fin rockets using the MDO methodology[J]. Canadian Aeronautics and Space Journal, 2010, 56(1): 17-30.

[3] 杨超, 黄超, 吴志刚, 等. 气动伺服弹性研究的进展与 挑战[J]. 航空学报, 2015, 36(4): 1011-1033.

YANG Chao, HUANG Chao, WU Zhigang, et al. Progress and challenges for aeroservoelasticity research[J]. 
Acta Aeronautica et Astronautica Sinica, 2015, 36(4): 1011-1033.

[4] 魏琼, 焦宗夏, 吴帅, 等. 气动伺服加载系统的非线性 复合控制[J]. 机械工程学报, 2017, 53(14): 217-224. WEI Qiong, JIAO Zongxia, WU Shuai, et al. Nonlinear compound control of pneumatic servo loading system[J]. Journal of Mechanical Engineering, 2017, 53(14) : 217-224

[5] VIDANOVIĆ N, RAŠUO B, KASTRATOVIĆ G, et al. Aerodynamic-structural missile fin optimization[J]. Aerospace Science and Technology, 2017, 65: 26-45.

[6] VERHAEGEN A, ŻBIKOWSKI R. Aeroservoelastic modelling and control of a slender anti-air missile for active damping of longitudinal bending vibrations[J]. Aerospace Science and Technology，2017，66: 20-27.

[7] 刘鸠云, 薛晓中, 孙瑞胜, 等. 曲面地表对远程火箭弹 弹道影响的计算方法 [J]. 火力与指挥控制, 2012,37(8): 44-46.

LIU Pengyun, XUE Xiaozhong, SUN Ruisheng, et al. A calculation method for influence of curved earth surface on far range rocket projectile calculating trajectory[J]. Fire Control and Command Control, 2012, 37(8): 44-46.

[8] 杨艳明, 唐胜景. 基于 Simulink 的子导弹全弹道仿真 [J]. 系统仿真学报, 2006, 18(6): 1442-1444.

YANG Yanming, TANG Shengjing. Trajectory simulation of submunitions based on simulink[J]. Journal of System Simulation, 2006, 18(6): 1442-1444.

[9] 杨希祥, 张为华. 小型固体运载火箭六自由度弹道仿真 [J]. 航空学报, 2010, 31(1): 41-47.

YANG Xixiang, ZHANG Weihua. Six-degree-of-freedom trajectory simulation of small solid launch vehicles[J]. Acta Aeronautica et Astronautica Sinica, 2010, 31(1): 41-47.

[10] 刘莉, 王岩松, 周思达, 等. 考虑弹体弹性的导弹半物 理仿真方法与影响分析 [J]. 北京航空航天大学学报, 2016, 42(4): 639-645.

LIU Li, WANG Yansong, ZHOU Sida, et al. Hardware-in-the-loop simulation method and influence analysis of missiles considering body elasticity[J]. Journal of Beijing University of Aeronautics and Astronautics, 2016, 42(4): 639-645.

[11] YAO S, WU Z G, YANG C. Trajectory simulation of flexible missiles and the effect of flexibility on hit precision[C]/AIAA Atmospheric Flight Mechanics Conference. National Harbor, Maryland, US: AIAA, 2014.

[12] SCHMIDT D K, RANEY D L. Modeling and simulation of flexible flight vehicles[J]. Journal of Guidance,
Control, and Dynamics, 2001, 24(3): 539-546.

[13] DU W, WIE B, WHORTON M. Dynamic modeling and flight control simulation of a large flexible launch vehicle[C]//AIAA Guidance, Navigation and Control Conference and Exhibit. Honolulu, Hawaii, US: AIAA, 2008.

[14] 董严, 付小燕, 张怀宇. 弹体结构变形对发射起始扰动 影响研究[J]. 弹箭与制导学报, 2016, 36(6): 75-77. DONG Yan, FU Xiaoyan, ZHANG Huaiyu. Study on effect of the projectile body's structural deformation to launching initial disturbance[J]. Journal of Projectile, Rockets, Missile and Guidance, 2016, 36(6): 75-77.

[15] RUSSKIKH S V. Vibrations of an elastic guide beam with a missile moving along it[J]. Russian Aeronautics, 2014, 57(1): 107-110.

[16] 杨超, 吴志刚. 导弹气动伺服弹性稳定性分析[J]. 飞行 力学, 2000, 18(4): 1-5.

YANG Chao, WU Zhigang. Aeroservoelastic stability of missile[J]. Flight Dynamics, 2000, 18(4): 1-5.

[17] WASZAK M, SCHMIDT D. Flight dynamics of aeroelastic vehicles[J]. Journal of Aircraft， 1988，25(6): 563-571.

[18] 徐劲祥. 弹道修正弹六自由度弹道仿真研究 [J]. 兵工 学报, 2007, 28(4): 411-413.

XU Jinxiang. Study on 6-DOF trajectory simulation of course correction projectiles[J]. Acta Armamentarii, 2007, 28(4): 411-413.

[19] HUA R H, ZHAO C X, YE Z Y, et al. Effect of elastic deformation on the trajectory of aerial separation $[\mathrm{J}]$. Aerospace Science and Technology, 2015, 45: 128-139.

[20] 陈克俊, 刘鲁华, 孟云鹤. 远程火箭飞行动力学与制导 [M]. 北京：国防工业出版社, 2014.

CHEN Kejun, LIU Luhua, MENG Yunhe. Launch vehicle flight dynamics and control[M]. Beijing : National Defense Industry Press, 2014.

[21] CAO R D, ZHANG X B. Multi-objective optimization of the aerodynamic shape of a long-range guided rocket[J]. Structural and Multidisciplinary Optimization, 2018, 57(4): 1779-1792.

[22] GHOSH S, YAKIMENKO O A, DAVIS D T, et al. Unmanned aerial vehicle guidance for an all-aspect approach to a stationary point[J]. Journal of Guidance, Control, and Dynamics, 2017, 40(11): 1-19.

作者简介: 魏钊, 男, 1994 年出生, 博士研究生。主要研究方向为飞行 器总体设计、飞行器气动弹性设计。

E-mail: weizhao@bit.edu.cn

李怀建 (通信作者), 男, 1973 年出生, 博士, 讲师。主要研究方向为飞 行器总体设计、卫星导航技术。

E-mail: lihuaijian@bit.edu.cn 